\title{
Generality of Proofs and its Brauerian Representation
}

\author{
Kosta DošEn and Zoran Petrić \\ Mathematical Institute, SANU \\ Knez Mihailova 35, p.f. 367 \\ 11001 Belgrade, Yugoslavia \\ email: \{kosta, zpetric\}@mi.sanu.ac.yu
}

\begin{abstract}
The generality of a derivation is an equivalence relation on the set of occurrences of variables in its premises and conclusion such that two occurrences of the same variable are in this relation if and only if they must remain occurrences of the same variable in every generalization of the derivation. The variables in question are propositional or of another type. A generalization of the derivation consists in diversifying variables without changing the rules of inference.

This paper examines in the setting of categorial proof theory the conjecture that two derivations with the same premises and conclusions stand for the same proof if and only if they have the same generality. For that purpose generality is defined within a category whose arrows are equivalence relations on finite ordinals, where composition is rather complicated. Several examples are given of deductive systems of derivations covering fragments of logic, with the associated map into the category of equivalence relations of generality.

This category is isomorphically represented in the category whose arrows are binary relations between finite ordinals, where composition is the usual simple composition of relations. This representation is related to a classical representation result of Richard Brauer.
\end{abstract}

Mathematics Subject Classification (2000): 03F07, 03G30, 18A15, 16 G99

Keywords: identity criteria for proofs, generality of proof, categories of proofs, Brauer algebras, representation 


\section{Introduction}

Up to now, two conjectures were made concerning identity criteria for proofs. The first, and dominating conjecture, which we call the Normalization Conjecture, was made by Prawitz in [17]. There Prawitz raised the question of identity criteria for proof as one of the central questions of general proof theory, and conjectured that two derivations in natural deduction should stand for the same proof iff they both reduce to the same normal form. (For details and further references concerning the Normalization Conjecture see [2].) Via the Curry-Howard correspondence, the Normalization Conjecture says that identity of proofs is caught by equality between typed lambda terms, and this shows that for fragments of intuitionistic logic the equivalence relation on derivations envisaged by Prawitz is a well-grounded mathematical notion. A further corroboration for this conjecture is brought by the categorial equivalence between typed lambda calculuses and cartesian closed categories, which was demonstrated by Lambek (see [14] and [15], Part I). Cartesian closed categories provide an alternative description of proofs in intuitionistic conjunctive-implicative logic, and the equivalence relation between proofs given by identity of arrows in cartesian closed categories is in accordance with the Normalization Conjecture.

The second conjecture concerning identity criteria for proofs, which we call the Generality Conjecture, was suggested by Lambek in [11] (pp. 287, 316) and [12] (p. 89). According to this conjecture, two derivations with the same premises and conclusions should stand for the same proof iff for every generalization of one of them, which consists in diversifying the variables without changing the rules of inference, there is a generalization of the other derivation so that in the two generalizations we have the same premises and conclusions. For example, let $\hat{k}_{p, p}^{1}: p \wedge p \rightarrow p$ and $\hat{k}_{p, p}^{2}: p \wedge p \rightarrow p$ be obtained respectively by the first rule and second rule of conjunction elimination, let $\left\langle\hat{k}_{p, p}^{1}, \hat{k}_{p, p}^{2}\right\rangle: p \wedge p \rightarrow p \wedge p$ be obtained from $\hat{k}_{p, p}^{1}$ and $\hat{k}_{p, p}^{2}$ by conjunction introduction, and let $1_{p \wedge p}: p \wedge p \rightarrow p \wedge p$ be the identity derivation from $p \wedge p$ to $p \wedge p$. For every generalization of $\left\langle\hat{k}_{p, p}^{1}, \hat{k}_{p, p}^{2}\right\rangle$ - and there is only one sort of generalization here, namely, $\left\langle\hat{k}_{q, r}^{1}, \hat{k}_{q, r}^{2}\right\rangle: q \wedge r \rightarrow q \wedge r$-we have a generalization of $1_{p \wedge p}$, namely, $1_{q \wedge r}: q \wedge r \rightarrow q \wedge r$. So, by the Generality Conjecture, $\left\langle\hat{k}_{p, p}^{1}, \hat{k}_{p, p}^{2}\right\rangle$ and $1_{p \wedge p}$ should stand for the same proof, which is in accordance with the Normalization Conjecture in its beta-eta version. On the other hand, $\hat{k}_{p, p}^{1}$ can be generalized to $\hat{k}_{q, r}^{1}: q \wedge r \rightarrow q$, and there is no generalization of $\hat{k}_{p, p}^{2}$ of the type $q \wedge r \rightarrow q$. So, by the Generality Conjecture, $\hat{k}_{p, p}^{1}$ and $\hat{k}_{p, p}^{2}$ would not stand for the same proof; this is again in accordance with the Normalization Conjecture. (We will see in Section 3 that when we go beyond the conjunctive fragment of logic, the two conjectures need not accord with each other any more.)

The notion of generality of derivation, which is implicit in the Generality Conjecture, will be made precise in the context of category theory. A proof will 
be an arrow in a category, and a derivation will be represented by an arrow term. The source of the arrow is the premise and the target the conclusion of the proof, and analogously for arrow terms and derivations. The generality of an arrow term will be an equivalence relation on the set of occurrences of variables in the source and target of this arrow term. If two occurrences in this set are equivalent, then they must be occurrences of the same variable (but not vice versa). The Generality Conjecture will say that two arrow terms with the same source and target stand for the same arrow iff they have the same generality.

Note that Lambek's formal notion of generality from [11] and [12], with which he became dissatisfied in [13] (p. 65), is not the notion we will define in this paper. We take from Lambek just the inspiration of his intuitive remarks about generality of proofs.

In the next section, we will introduce a category called Gen, in which we will define formally the generality of derivations. We may conceive this category as obtained by abstraction from the category whose arrows are proofs and whose objects are formulae by replacing formulae with sequences of occurrences of variables. From arrows that stood for proofs we keep only the equivalence relation holding between occurrences of variables that must in every generalization remain occurrences of the same variable. Since sequences of occurrences of variables are finite we represent them by finite ordinals. The variables that motivate the introduction of Gen are of an arbitrary type: they may be propositional variables, or individual variables, or of any other type of variable in a language from which we take our formulae. We may also deal simultaneously with several types of variable.

In Section 3, we will give several examples of deductive systems, covering fragments of logic, and of generality obtained via maps into Gen defined for these deductive systems, which induces identity of proofs according to the Generality Conjecture.

The definition of composition of arrows in Gen is rather involved, and we will not attempt to give a direct formal proof of the associativity of this composition. An indirect proof of this associativity will be provided by an isomorphic representation of Gen in the category Rel whose arrows are binary relations between finite ordinals, where composition is just composition of relations. We devote Sections 4-6 to this matter. In Section 4 we first deal with some very general questions about representing equivalence relations, while in Section 5 we give our representation of Gen in Rel, and prove the isomorphism of this representation. In Section 6 we show that our representation of Gen in Rel is related to a classical result of representation theory due to Richard Brauer.

The representation of Gen in Rel does not serve only to prove the associativity of the composition of Gen, but it may be of an independent interest. Although in passing from Gen to Rel we have a functor involving an exponential function, Rel may be helpful in computations, since composing binary relations is like multiplying matrices. The representation of Gen in Rel sheds also some light on Brauer's representation result, which it generalizes to a certain extent. 
In a companion to this paper [6] we will consider a category more general than Gen obtained by replacing equivalence relations by preordering, i.e. reflexive and transitive, relations. This category will also be isomorphically represented in Rel, in a manner that generalizes further Brauer's representation.

\section{The category Gen}

The objects of the category Gen are finite ordinals, and its arrows $R: n \rightarrow m$ are equivalence relations $R \subseteq(n+m)^{2}$. As usual, we write sometimes $x R y$ for $(x, y) \in R$.

If $X \subseteq \omega$, then $X^{+k}={ }_{\text {def }}\{n+k \mid n \in X\}$. For $n, m \in \omega$, the sum $n+m$ is by definition $n \cup m^{+n}$. For an arbitrary relation $R \subseteq\left(n \cup m^{+n}\right)^{2}$ and $k \in \omega$, let $R^{+k} \subseteq\left(n^{+k} \cup m^{+n+k}\right)^{2}$ be the relation defined by

$$
x R^{+k} y \quad \text { iff } \quad(x-k) R(y-k) .
$$

Note that if $R$ is an equivalence relation in Gen, then for $k \neq 0$ the equivalence relation $R^{+k}$ is not an arrow of Gen.

The identity arrow $1_{n}$ in Gen is the equivalence relation $1_{n} \subseteq(n+n)^{2}$ defined by

$$
(x, y) \in 1_{n} \quad \text { iff } \quad x=y(\bmod n) .
$$

We define composition of arrows in Gen in the following manner. Let $\mathrm{Cl}(R)$ be the transitive closure of the binary relation $R$. Suppose we have the equivalence relations $R_{1}: n \rightarrow m$ and $R_{2}: m \rightarrow k$; that is, $R_{1} \subseteq\left(n \cup m^{+n}\right)^{2}$ and $R_{2} \subseteq\left(m \cup k^{+m}\right)^{2}$. Then $R_{1} \cup R_{2}^{+n} \subseteq\left(n \cup m^{+n} \cup k^{+m+n}\right)^{2}$, and it is easy to check that $\mathrm{Cl}\left(R_{1} \cup R_{2}^{+n}\right)$ is an equivalence relation.

For $x \in n \cup k^{+n}$ and $m \in \omega$ let

$$
x^{\prime}=\left\{\begin{array}{lll}
x & \text { if } & x \in n \\
x+m & \text { if } & x \in k^{+n}
\end{array}\right.
$$

Then for an arbitrary relation $R \subseteq\left(n \cup k^{+m+n}\right)^{2}$ let $R^{\dagger} \subseteq\left(n \cup k^{+n}\right)^{2}$ be defined by

$$
x R^{\dagger} y \quad \text { iff } \quad x^{\prime} R y^{\prime} .
$$

It is easy to see that if $R$ is an equivalence relation, then so is $R^{\dagger}$.

Then we define the composition $R_{2} * R_{1}: n \rightarrow k$ of the arrows $R_{1}$ and $R_{2}$ above by

$$
R_{2} * R_{1}={ }_{\text {def }}\left(\left(\mathrm{Cl}\left(R_{1} \cup R_{2}^{+n}\right) \cap\left(n \cup k^{+m+n}\right)^{2}\right)^{\dagger} .\right.
$$

It can be checked that $R_{2} * R_{1}$ is indeed an equivalence relation on $n \cup k^{+n}$. It is more involved to check formally that $*$ is associative, though this is rather clear if we represent this operation geometrically (as this is done in [7], and also in 
categories of tangles; see [9], Chapter 12, and references therein). For example, for $R_{1} \subseteq\left(3 \cup 9^{+3}\right)^{2}=12^{2}$ that corresponds to the partition $\{\{0,3\},\{4,5\}$, $\{1,6\},\{7,8\},\{2,9\},\{10,11\}\}$, and $R_{2} \subseteq\left(9 \cup 1^{+9}\right)^{2}=10^{2}$ that corresponds to the partition $\{\{0,1\},\{2,9\},\{3,4\},\{5,6\},\{7,8\}\}$, the composition $R_{2} * R_{1} \subseteq$ $\left(3 \cup 1^{+3}\right)^{2}=4^{2}$ that corresponds to the partition $\{\{0,3\},\{1,2\}\}$ is obtained from the following drawing

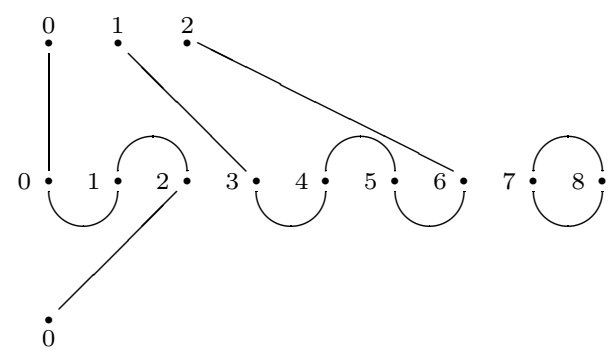

In this example all members of partitions have two elements, but this is by no means necessary.

The complexity of the definition of $*$ in Gen motivates the introduction of an isomorphic representation of Gen, considered in Section 5, in which composition will be defined in an elementary way. The proof that we have there an isomorphic representation will provide an indirect proof that $*$ in Gen is associative, and that Gen is indeed a category.

\section{Generality of derivations in fragments of logic}

The language $\mathcal{L}$ of conjunctive logic is built from a nonempty set of propositional variables $\mathcal{P}$ with the binary connective $\wedge$ and the propositional constant, i.e. nullary connective, $T$ (the exact cardinality of $\mathcal{P}$ is not important here). We use the schematic letters $A, B, C, \ldots$ for formulae of $\mathcal{L}$.

We have the following axiomatic derivations for every $A$ and $B$ in $\mathcal{L}$ :

$$
\begin{aligned}
& 1_{A}: A \rightarrow A, \\
& \hat{k}_{A, B}^{1}: A \wedge B \rightarrow A, \\
& \hat{k}_{A, B}^{2}: A \wedge B \rightarrow B, \\
& \hat{k}_{A}: A \rightarrow \top,
\end{aligned}
$$

and the following inference rules for generating derivations:

$$
\frac{f: A \rightarrow B \quad g: B \rightarrow C}{g \circ f: A \rightarrow C}
$$




$$
\frac{f: C \rightarrow A \quad g: C \rightarrow B}{\langle f, g\rangle: C \rightarrow A \wedge B}
$$

This defines the deductive system $\mathcal{D}$ of conjunctive logic (both intuitionistic and classical). In this system $T$ is included as an "empty conjunction". For $f: A \rightarrow$ $B$ and $g: C \rightarrow D$ let $f \wedge g: A \wedge C \rightarrow B \wedge D$ abbreviate $\left\langle f \circ \hat{k}_{A, C}^{1}, g \circ \hat{k}_{A, C}^{2}\right\rangle$.

We define now a function $G$ from $\mathcal{L}$ to the objects of Gen by taking that $G(A)$ is the number of occurrences of propositional variables in $A$. Next we define inductively a function, also denoted by $G$, from the derivations of $\mathcal{D}$ to the arrows of Gen:

$$
\begin{aligned}
& G\left(1_{A}\right)=1_{G(A)}, \\
& (x, y) \in G\left(\hat{k}_{A, B}^{1}\right) \quad \text { iff } \quad x=y(\bmod G(A \wedge B)), \\
& (x, y) \in G\left(\hat{k}_{A, B}^{2}\right) \quad \text { iff } \quad(x, y<G(A) \text { and } x=y) \text { or }(x, y \geq G(A) \text { and } \\
& x=y(\bmod G(B))) \text {, } \\
& (x, y) \in G\left(\hat{k}_{A}\right) \quad \text { iff } \quad x=y, \\
& G(g \circ f)=G(g) * G(f) \text {; } \\
& \text { for } x \in G(C) \cup G(B)^{+G(A)+G(C)} \text { let } \\
& x^{\prime \prime}= \begin{cases}x & \text { if } x \in G(C) \\
x-G(A) & \text { if } x \in G(B)^{+G(A)+G(C)},\end{cases}
\end{aligned}
$$

and let $(x, y) \in G(g)^{\dagger \dagger}$ iff $\left(x^{\prime \prime}, y^{\prime \prime}\right) \in G(g)$; then

$$
G(\langle f, g\rangle)=\operatorname{Cl}\left(G(f) \cup G(g)^{\dagger \dagger}\right) .
$$

Then, in accordance with the Generality Conjecture, we stipulate that two derivations $f, g: A \rightarrow B$ of $\mathcal{D}$ are equivalent iff $G(f)=G(g)$. A proof of $\mathcal{D}$ is then the equivalence class of a derivation with respect to this equivalence relation.

It is then clear that proofs make the arrows of a category $\mathcal{C}$, with the obvious sources and targets, and that $G$ gives rise to a faithful functor from $\mathcal{C}$ to Gen. The category $\mathcal{C}$ happens to be the free cartesian category generated by the set of propositional variables $\mathcal{P}$ as the generating set of objects (this set may be conceived as a discrete category). This fact about $\mathcal{C}$ follows from the coherence result for cartesian categories treated in [4] and [16]. (Cartesian categories are categories with all finite products, including the empty product, i.e. terminal object. The category $\mathcal{C}$ can be equationally presented; see [15], Chapter I.3, or [4].)

The language of disjunctive logic is dual to the language we had above: instead of $\wedge$ and $T$ we have $\vee$ and $\perp$ in $\mathcal{L}$, and instead of $\hat{k}^{i}, \hat{k}$ and $\langle$,$\rangle we$ 
have in the corresponding deductive system $\mathcal{D}$

$$
\begin{gathered}
\stackrel{\vee}{*}_{A, B}^{1}: A \rightarrow A \vee B, \\
\breve{k}_{A, B}^{2}: B \rightarrow A \vee B, \\
\check{k}_{A}: \perp \rightarrow A, \\
\frac{f: A \rightarrow C \quad g: B \rightarrow C}{[f, g]: A \vee B \rightarrow C}
\end{gathered}
$$

Dually to what we had above, we obtain that equivalence of derivations induced by generality makes of $\mathcal{D}$ the free category with all finite coproducts generated by $\mathcal{P}$.

Let us now assume we have in $\mathcal{D}$ both $\wedge$ and $\vee$, but without $T$ and $\perp$, and let us introduce the category of proofs $\mathcal{C}$ induced by generality as we did above. The category $\mathcal{C}$ will, however, not be the free category with nonempty finite products and coproducts generated by $\mathcal{P}$, in spite of the coherence result of [5]. The problem is that in $\mathcal{C}$ we don't have the equation

$$
\hat{k}_{A, B}^{1} \circ\left(1_{A} \wedge\left[1_{B}, 1_{B}\right]\right)=\hat{k}_{A, B \vee B}^{1} .
$$

The graphs with respect to which coherence is proved in [5] don't correspond to the equivalence relations of Gen. The graphs of [4], with respect to which coherence can be proved for cartesian categories are also not the equivalence relations of Gen, but in this case one easily passes to Gen from the category whose arrows are these graphs.

The coherence result of [10] for symmetric monoidal closed categories without the unit object I can be used to show that generality in the appropriate deductive system, which corresponds to the implication-tensor fragment of intuitionistic linear logic, gives rise to the free symmetric monoidal closed category without I. The graphs of [10] correspond to the equivalence relations of Gen.

If we add intuitionistic implication to conjunctive or conjunctive-disjunctive logic, the equivalence of derivations induced by generality does not give rise to free cartesian closed or free bicartesian closed categories (for counterexamples see [5], Section 1, and [18]).

Up to now, we considered generality of derivations in fragments of logic with respect to propositional variables. In the next example this generality will be considered with respect to individual variables.

Let now $\mathcal{L}$ be the language generated from a nonempty set of individual variables $\{x, y, z, \ldots\}$, whose exact cardinality is again not important, with the binary relational symbol $=$, the binary connective $\wedge$ and the propositional constant $T$.

As axioms and inference rules for our deductive system $\mathcal{D}$ we now have whatever we had for conjunctive logic in the preceding section plus all axioms of the form 


$$
\begin{aligned}
& r_{x}: \top \rightarrow x=x \\
& s_{x, y}: x=y \rightarrow y=x \\
& t_{x, y, z}: x=y \wedge y=z \rightarrow x=z
\end{aligned}
$$

The function $G$ from $\mathcal{L}$ to the objects of Gen is now defined by taking that $G(A)$ is the number of occurrences of individual variables in $A$. We define inductively the function $G$ from the derivations of $\mathcal{D}$ to the arrows of $G e n$ as we did in the preceding section for conjunctive logic with the following additional clauses, where $X^{+}$is the reflexive and symmetric closure of $X$,

$$
\begin{aligned}
& G\left(r_{x}\right)=\{(0,1)\}^{+}, \\
& G\left(s_{x, y}\right)=\{(0,3),(1,2)\}^{+}, \\
& G\left(t_{x, y, z}\right)=\{(0,4),(1,2),(3,5)\}^{+} .
\end{aligned}
$$

Graphically, these clauses correspond to

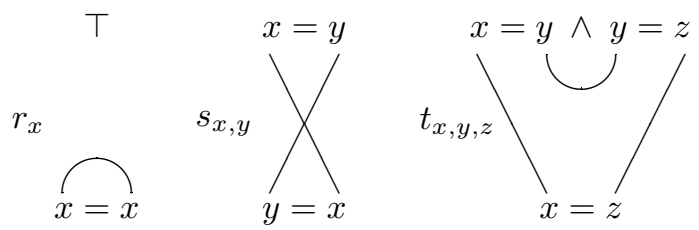

Then in the category $\mathcal{C}$ of proofs defined via generality we find, for example, the following equations:

$$
\begin{aligned}
& t_{x, y, y} \circ\left(1_{x=y} \wedge r_{y}\right)=\hat{k}_{x=y, \top}^{1}, \\
& t_{x, x, y} \circ\left(r_{x} \wedge 1_{x=y}\right)=\hat{k}_{\top, x=y}^{2}, \\
& s_{y, x} \circ s_{x, y}=1_{x=y}, \\
& s_{x, x} \circ r_{x}=r_{x} .
\end{aligned}
$$

\section{Representing equivalence relations by sets of functions}

In this section we will consider some general matters concerning the representation of arbitrary equivalence relations. This will serve for demonstrating in the next section the isomorphism of our representation of Gen in the category whose arrows are binary relations between finite ordinals.

Let $X$ be an arbitrary set, and let $R \subseteq X^{2}$. Let $p$ be a set such that for $p_{0} \neq p_{1}$ we have $p_{0}, p_{1} \in p$. Let $S \subseteq p^{2}$, and for $i, j \in\{0,1\}$ let $p_{i} S p_{j}$ iff $i \leq j$. Consider the following set of functions:

$$
\mathcal{F}^{S}(R)=_{\text {def }}\{f: X \rightarrow p \mid(\forall x, y \in X)(x R y \Rightarrow(f(x), f(y)) \in S)\},
$$


and for $x, y \in X$ let $f_{x}: X \rightarrow p$ be defined as follows:

$$
f_{x}(y)= \begin{cases}p_{1} & \text { if } x R y \\ p_{0} & \text { if not } x R y .\end{cases}
$$

The function $f_{x}$ is the characteristic function of the $R$-cone over $x$. We can then prove the following proposition.

Proposition 1. The relation $R$ is transitive iff $(\forall x \in X) f_{x} \in \mathcal{F}^{S}(R)$.

Proof. $\quad(\Rightarrow)$ Suppose $y R z$. If $f_{x}(y)=p_{0}$, then $\left(f_{x}(y), f_{x}(z)\right) \in S$. If $f_{x}(y)=p_{1}$, then $f_{x}(z)=p_{1}$ by the transitivity of $R$.

$(\Leftarrow)$ If $y R z \Rightarrow\left(f_{x}(y), f_{x}(z)\right) \in S$, then $y R z \Rightarrow\left(f_{x}(y)=p_{0}\right.$ or $\left.f_{x}(z)=p_{1}\right)$, which means $y R z \Rightarrow(x R y \Rightarrow x R z)$.

With the definition

$$
\mathcal{F}^{=}(R)=_{\text {def }}\{f: X \rightarrow p \mid(\forall x, y \in X)(x R y \Rightarrow f(x)=f(y))\}
$$

we can prove the following proposition.

Proposition 2. If $S$ is reflexive and antisymmetric, and $R$ is symmetric, then $\mathcal{F}^{=}(R)=\mathcal{F}^{S}(R)$.

Proof. If $f \in \mathcal{F}^{=}(R)$, then $f \in \mathcal{F}^{S}(R)$ by the reflexivity of $S$; and if $f \in \mathcal{F}^{S}(R)$, then $f \in \mathcal{F}^{=}(R)$ by the symmetry of $R$ and the antisymmetry of $S$.

Suppose now the relation $S$ is reflexive and antisymmetric. We can then prove the following proposition.

Proposition 3. The relation $R$ is an equivalence relation iff $(\forall x, y \in X)(x R y$ $\left.\Leftrightarrow\left(\forall f \in \mathcal{F}^{=}(R)\right) f(x)=f(y)\right)$.

Proof. Note first that in the equivalence on the right-hand side the direction $x R y \Rightarrow\left(\forall f \in \mathcal{F}^{=}(R)\right) f(x)=f(y)$ is satisfied by definition.

$(\Rightarrow)$ If $\left(\forall f \in \mathcal{F}^{=}(R)\right) f(x)=f(y)$, then $f_{x}(x)=f_{x}(y)$ by Propositions 1 and 2 , and since $f_{x}(x)=p_{1}$ by the reflexivity of $R$, we have $x R y$.

$(\Leftarrow)$ From

$$
(*) \quad(\forall x, y \in X)\left(\left(\forall f \in \mathcal{F}^{=}(R)\right) f(x)=f(y) \Rightarrow x R y\right)
$$

we obtain that $R$ is reflexive by taking that $x=y$.

For transitivity, suppose $x R y$ and $y R z$. Then for every $f \in \mathcal{F}=(R)$ we have $f(x)=f(y)=f(z)$, and hence $x R z$ by $(*)$.

For symmetry, suppose $x R y$. Then for every $f \in \mathcal{F}^{=}(R)$ we have $f(y)=$ $f(x)$, and hence $y R x$ by $(*)$.

COROLlary. If $R_{1}, R_{2} \subseteq X^{2}$ are equivalence relations, then $R_{1}=R_{2}$ iff $\mathcal{F}=\left(R_{1}\right)=\mathcal{F}=\left(R_{2}\right)$. 
Proof. We have, of course, that $R_{1}=R_{2}$ implies $\mathcal{F}=\left(R_{1}\right)=\mathcal{F}=\left(R_{2}\right)$. The converse follows from Proposition 3.

\section{Representing Gen in the category Rel}

Let $R e l$ be the category whose arrows are binary relations between finite ordinals. Let $I_{n} \subseteq n \times n$ be the identity relation on $n$; the composition $R_{2} \circ R_{1} \subseteq n \times k$ of $R_{1} \subseteq n \times m$ and $R_{2} \subseteq m \times k$ is $\left\{(x, y) \mid(\exists z \in m)\left(x R_{1} z\right.\right.$ and $\left.\left.z R_{2} y\right)\right\}$.

Then for $p \in \omega$ such that $p \geq 2$ we define a functor $F_{p}$ from Gen to Rel in the following manner. On objects $F_{p}$ is defined by $F_{p}(n)=p^{n}$. Every element of the ordinal $p^{n}$ is identified by a function $f: n \rightarrow p$ in the following way. Every $f: n \rightarrow p$ corresponds to a sequence of members of $p$ of length $n$. These sequences can be ordered lexicographically, and $\left(p^{n}, \leq\right)$ is isomorphic to the set of these sequences ordered lexicographically.

For $f_{1}: n \rightarrow p$ and $f_{2}: m \rightarrow p$, let $\left[f_{1}, f_{2}\right]: n \cup m^{+n} \rightarrow p$ be defined by

$$
\left[f_{1}, f_{2}\right](x)=\left\{\begin{array}{ll}
f_{1}(x) & \text { if } x \in n \\
f_{2}(x-n) & \text { if } x \in m^{+n}
\end{array} .\right.
$$

For $R: n \rightarrow m$ an arrow of Gen, and for $f_{1}: n \rightarrow p$ and $f_{2}: m \rightarrow p$, we define $F_{p}(R)$ by

$$
\left(f_{1}, f_{2}\right) \in F_{p}(R) \quad \text { iff } \quad\left[f_{1}, f_{2}\right] \in \mathcal{F}^{=}(R),
$$

where $\mathcal{F}=(R)$ is the set of functions defined as in the preceding section. Here $X$ is $n+m$, while for the ordinal $p \geq 2$ we have that $p_{0}$ is $0, p_{1}$ is 1 and $S$ is $\leq$. It remains to check that $F_{p}$ so defined is indeed a functor.

Proposition 4. $F_{p}$ is a functor.

Proof. We show first that $F_{p}\left(1_{n}\right)=I_{p^{n}}$ by remarking that

$$
\left[f_{1}, f_{2}\right] \in \mathcal{F}^{=}\left(1_{n}\right) \quad \text { iff } \quad f_{1}=f_{2} .
$$

Next we have to show that $F_{p}\left(R_{2} * R_{1}\right)=F_{p}\left(R_{2}\right) \circ F_{p}\left(R_{1}\right)$ for $R_{1}: n \rightarrow m$ and $R_{2}: m \rightarrow k$. This amounts to showing that for $f_{1}: n \rightarrow p$ and $f_{2}: k \rightarrow p$

$$
(* *) \quad\left(\forall x, y \in n \cup k^{+n}\right)\left(x\left(R_{2} * R_{1}\right) y \Rightarrow\left[f_{1}, f_{2}\right](x)=\left[f_{1}, f_{2}\right](y)\right)
$$

is equivalent to the assertion that there exists an $f_{3}: m \rightarrow p$ such that the following two statements are satisfied:

$$
\begin{aligned}
& (* 1) \quad\left(\forall x, y \in n \cup m^{+n}\right)\left(x R_{1} y \Rightarrow\left[f_{1}, f_{3}\right](x)=\left[f_{1}, f_{3}\right](y)\right), \\
& (* 2) \quad\left(\forall x, y \in m \cup k^{+m}\right)\left(x R_{2} y \Rightarrow\left[f_{3}, f_{2}\right](x)=\left[f_{3}, f_{2}\right](y)\right) .
\end{aligned}
$$

First we show that $(* *)$ implies that for some $f_{3}: m \rightarrow p$ we have $(* 1)$ and $(* 2)$. Before defining $f_{3}$ we check the following facts that follow from $(* *)$ : 


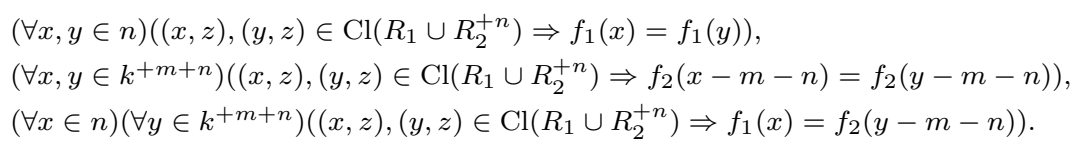

Then for $z \in m$ we define $f_{3}(z) \in p$ as follows. If for some $x \in n \cup k^{+m+n}$ we have $(x, z+n) \in \mathrm{Cl}\left(R_{1} \cup R_{2}^{+n}\right)$, then

$$
f_{3}(z)= \begin{cases}f_{1}(x) & \text { if } x \in n \\ f_{2}(x-m-n) & \text { if } x \in k^{+m+n} .\end{cases}
$$

This definition is correct according to (I)-(III). Otherwise, if there is no such $x$, we put $f_{3}(z)=0$.

Let us now demonstrate $(* 1)$. Suppose $x R_{1} y$. If $x, y \in n$, then $f_{1}(x)=f_{1}(y)$ by $(* *)$. If $x, y \in m^{+n}$, then $f_{3}(x-n)=f_{3}(y-n)$ by the definition of $f_{3}$. If $x \in n$ and $y \in m^{+n}$, then $f_{1}(x)=f_{3}(y-n)$ by the definition of $f_{3}$. In every case we obtain $\left[f_{1}, f_{3}\right](x)=\left[f_{1}, f_{3}\right](y)$. We demonstrate analogously $(* 2)$.

It remains to show that from the assumption that for some $f_{3}: m \rightarrow p$ we have $(* 1)$ and $(* 2)$ we can infer $(* *)$. Suppose $x\left(R_{2} * R_{1}\right) y$ and $x, y \in n$. Then there is a sequence $x_{1}, \ldots, x_{2 l}, l \geq 1$, such that $x=x_{1}, y=x_{2 l}$, for every $i$ such that $1<i<2 l$ we have $x_{i} \in m^{+n}$, and

$$
x_{1} R_{1} x_{2}, x_{2} R_{2}^{+n} x_{3}, x_{3} R_{1} x_{4}, \ldots, x_{2 l-1} R_{1} x_{2 l} .
$$

Then by applying $(* 1)$ and $(* 2)$ we obtain $f_{1}(x)=f_{1}(y)$, and hence $\left[f_{1}, f_{2}\right](x)=$ $\left[f_{1}, f_{2}\right](y)$. We proceed analogously when $x, y \in k^{+n}$, or when $x \in n$ and $y \in k^{+n}$.

Next we show that $F_{p}$ is faithful. Since $F_{p}$ is one-one on objects, this amounts to showing that it is one-one on arrows.

Proposition 5. $F_{p}$ is faithful.

Proof. Suppose $F_{p}\left(R_{1}\right)=F_{p}\left(R_{2}\right)$. This means that for every $f_{1}: n \rightarrow p$ and every $f_{2}: m \rightarrow p$ we have $\left[f_{1}, f_{2}\right] \in \mathcal{F}=\left(R_{1}\right)$ iff $\left[f_{1}, f_{2}\right] \in \mathcal{F}=\left(R_{2}\right)$. But every function $f: n+m \rightarrow p$ is of the form $\left[f_{1}, f_{2}\right]$ for some $f_{1}: n \rightarrow p$ and some $f_{2}: m \rightarrow p$. Hence $\mathcal{F}^{=}\left(R_{1}\right)=\mathcal{F}^{=}\left(R_{2}\right)$, and $R_{1}=R_{2}$ by the Corollary of the preceding section.

So we have an isomorphic representation of Gen in Rel.

\section{Connection with Brauer algebras}

The representation of the category Gen in the category Rel, which we have presented in the preceding section, is closely connected to Brauer's representation of Brauer algebras, which is the orthogonal group case of [1] (Section 5; see also [19], Section 2, [8], Section 3, and [3]). 
An $(n, n)$-diagram is an arrow $R: n \rightarrow n$ of $G e n$ such that every member of the partition that corresponds to $R$ is a two-element set. When defining the composition $R_{2} * R_{1}: n \rightarrow k$ of $R_{1}: n \rightarrow m$ and $R_{2}: m \rightarrow k$ we didn't take into account the "circles", or "closed loops", in $\mathrm{Cl}\left(R_{1} \cup R_{2}^{+n}\right)$, namely, those members $X$ of the partition corresponding to $\mathrm{Cl}\left(R_{1} \cup R_{2}^{+n}\right)$ such that $X \cap n=\emptyset$ and $X \cap k^{+m+n}=\emptyset$. (In our example in Section 2 we have a circle involving 7 and 8 in the drawing.) Let $l\left(R_{1}, R_{2}\right)$ be the number of those circles.

For $n \in \omega$ and $c$ a complex number, the Brauer algebra $B(n, c)$ is the algebra with basis the set of all $(n, n)$-diagrams, and multiplication between two $(n, n)$ diagrams $R_{1}$ and $R_{2}$ defined as $c^{l\left(R_{1}, R_{2}\right)}\left(R_{2} * R_{1}\right)$ (cf. [8], Section 2).

Let $V$ be a vector space of dimension $p$ with basis $w_{0}, \ldots, w_{p-1}$. For the $(n, n)$-diagram $R$ define $\beta(R) \in \operatorname{End}\left(\otimes^{n} V\right)$ by the matrix (with respect to the basis $\left\{w_{a_{0}} \otimes \ldots \otimes w_{a_{n-1}} \mid a_{i} \in\{0, \ldots, p-1\}\right\}$ of $\left.\otimes^{n} V\right)$

$$
\beta(R)_{a_{0} \ldots a_{n-1}, a_{n} \ldots a_{2 n-1}}=\operatorname{def} \prod_{\{i, j\} \in R} \delta\left(a_{i}, a_{j}\right)
$$

where $\delta$ is the Kronecker $\delta$ (cf. [8], Definition 3.1). The sequences $a_{0} \ldots a_{n-1}$ and $a_{n} \ldots a_{2 n-1}$ correspond to functions from $n$ to $p$. This defines a homomorphism of the Brauer algebra $B(n, p)$ onto a subalgebra of $\operatorname{End}\left(\otimes^{n} V\right)$ (see [8], Lemma 3.2 ), which is Brauer's representation of Brauer algebras in [1] (Section 5).

Every $n \times m$-matrix $M$ whose entries are only 0 and 1 may be identified with a binary relation $\mathcal{R}_{M} \subseteq n \times m$ such that $M(i, j)=1$ iff $(i, j) \in \mathcal{R}_{M}$. The matrix $M$ is the characteristic function of $\mathcal{R}_{M}$. The $p^{n} \times p^{n}$-matrix $\beta(R)$ is a 0-1 matrix such that $\mathcal{R}_{\beta(R)}$ is precisely $F_{p}(R)$. So at the root of Brauer's representation we find a particular case of our representation of Gen in Rel.

One difference between our representation and Brauer's is that we deal with arbitrary equivalence relations, whereas Brauer's equivalence relations are more special - they correspond to partitions whose every member is a two-element set. Another difference is that we have composition of relations where Brauer has multiplication of matrices. One passes from multiplication of matrices to our composition of relations by disregarding "circles" and by taking that $1+1=1$.

Acknowledgement. The writing of this paper was financed by the Ministry of Science, Technology and Development of Serbia through grant 1630 (Representation of proofs with applications, classification of structures and infinite combinatorics).

Acknowledgement added on 8 April 2016. We would like to thank Sonja Telebaković for helping us to correct in this [v7] version of our paper a mistake concerning the function $f_{3}$ (in the definition of this function and in the demonstration of $(* 1)$ in the first paragraph after this definition) in Section 5 of the previous versions of the paper. 


\section{References}

[1] R. Brauer, On algebras which are connected with the semisimple continuous groups, Ann. of Math. 38 (1937), pp. 857-872.

[2] K. Došen, Identity of proofs based on normalization and generality, 2002 (available at: http:// arXiv. org/ math. LO/ 0208094).

[3] K. Došen, Ž. Kovijanić and Z. Petrić, A new proof of the faithfulness of Brauer's representation of Temperley-Lieb algebras, 2002 (available at: http:// arXiv. org/ math. GT/ 0204214).

[4] K. Došen and Z. Petrić, The maximality of cartesian categories, Math. Logic Quart. 47 (2001), pp. 137-144 (available at: http:// arXiv. org/ math. CT/ 9911059).

[5] K. Došen and Z. Petrić, Bicartesian coherence, Studia Logica 71 (2002), pp. 331-353 (available at: http:// arXiv. org/ math. CT/ 0006052).

[6] K. Došen and Z. Petrić, A Brauerian representation of split preorders, 2002 (available at: http:// arXiv. org/ math. LO/ 00211277).

[7] S. Eilenberg and G.M. Kelly, A generalization of the functorial calculus, J. Algebra 3 (1966), pp. 366-375.

[8] V.F.R. Jones, A quotient of the affine Hecke algebra in the Brauer algebra, Enseign. Math. (2) 40 (1994), pp. 313-344.

[9] C. Kassel, Quantum Groups, Springer, Berlin, 1995.

[10] G.M. Kelly and S. Mac Lane, Coherence in closed categories, J. Pure Appl. Algebra 1 (1971), pp. 97-140, 219.

[11] J. Lambek, Deductive systems and categories I: Syntactic calculus and residuated categories, Math. Systems Theory 2 (1968), pp. 287-318.

[12] J. Lambek, Deductive systems and categories II: Standard constructions and closed categories, in: Category Theory, Homology Theory and their Applications I, Lecture Notes in Math. 86, Springer, Berlin, 1969, pp. 76122.

[13] J. Lambek, Deductive systems and categories III: Cartesian closed categories, intuitionist propositional calculus, and combinatory logic, in: F.W. Lawvere ed., Toposes, Algebraic Geometry and Logic, Lecture Notes in Math. 274, Springer, Berlin, 1972, pp. 57-82.

[14] J. Lambek, Functional completeness of cartesian categories, Ann. Math. Logic 6 (1974), pp. 259-292. 
[15] J. Lambek and P.J. Scott, Introduction to Higher-Order Categorical Logic, Cambridge University Press, Cambridge, 1986.

[16] Z. Petrić, Coherence in substructural categories, Studia Logica 70 (2002), pp. 271-296 (available at: http:// arXiv. org/ math. CT/ 0006061).

[17] D. Prawitz, Ideas and results in proof theory, in: J.E. Fenstad ed., Proceedings of the Second Scandinavian Logic Symposium, North-Holland, Amsterdam, 1971, pp. 235-307.

[18] M.E. Szabo, A counter-example to coherence in cartesian closed categories, Canad. Math. Bull. 18 (1975), pp. 111-114.

[19] H. Wenzl, On the structure of Brauer's centralizer algebras, Ann. of Math. 128 (1988), pp. 173-193. 